\title{
Estresse e Distresse: Estudo com a Escala de Faces em Aracaju (SE)
}

\author{
André Faro ${ }^{1}$ \\ Departamento de Psicologia da Universidade Federal de Sergipe, São Cristóvão, SE, Brasil
}

\section{Resumo}

A presente pesquisa objetivou levantar dados a respeito da autopercepção de estresse em Aracaju (SE), como também, identificar a presença de distresse, caracterizar o perfil sociodemográfico e dos hábitos de saúde da amostra, além de mapear relações desses perfis quanto à variabilidade do estresse e do distresse. A amostragem foi realizada por meio da técnica do ponto-de-fluxo, em que participaram 2135 transeuntes adultos que se encontravam no principal centro comercial de Aracaju (SE). Utilizou-se um questionário para caracterização sociodemográfica e de hábitos de saúde, enquanto que o estresse e o distresse foram mensurados com a Escala de Faces (sete pontos). Para a análise estatística foi estimado o impacto das variáveis sobre o estresse, por meio de regressão linear, e sobre o distresse, com regressão logística. Quanto aos resultados, $15,1 \%$ dos participantes apresentaram distresse e a média na escala de faces foi de três pontos (Desvio-Padrão = 1,5). As variáveis renda, sexo, tabagismo e prática de atividade física exibiram significância estatística em relação ao estresse. Nas modelagens por regressão linear e logística, as variáveis com impacto aditivo sobre o estresse foram o tabagismo e o sexo feminino, e com impacto subtrativo a renda média familiar (igual ou acima de dez salários mínimos) e a prática de atividade física regular. Discutiram-se, além dos modelos, características da medida do estresse e diferenças observadas nas análises via regressão linear e logística. Ao final, apontaram-se limitações do estudo, especialmente quanto à escolha da Escala de Faces, como também, foram sugeridas novas possibilidades de pesquisa.

Palavras-chave: Estresse, distresse, escala de faces, perfil sociodemográfico, hábitos de saúde.

\section{Stress and Distress: Study with Faces Scale in Aracaju (SE)}

\begin{abstract}
This research aimed to collect data about self-perception of stress in Aracaju (SE), as well as to identify the presence of distress, to characterize the sociodemographic profile and health habits of the sample and map out relationships of these profiles on the variability of stress and distress. The sampling was performed by flow point, with 2135 adults that were in the main commercial center of Aracaju (SE). It used a questionnaire for characterization the sociodemographic and health profiles, whereas the stress and distress were measured by Faces Scale (seven points). For multivariate statistical analysis, it estimated the impact of sociodemographic and health variables on stress by linear regression, and on distress with logistic regression. Concerning the results, $15.1 \%$ of participants showed distress and the mean of the faces scale was three points (standard deviation $=1.5$ ). The variables income, gender, to smoke and physical activity practice showed statistical significance in relation to stress. In modeling by linear and

Endereço para correspondência: Departamento de Psicologia. Universidade Federal de Sergipe, Av. Marechal Rondon, s/n, Conjunto Rosa Elze, São Cristóvão, SE, Brasil 49000-000. Fone: 79 8103-6627. E-mail: andre. faro.ufs@gmail.com

Agradecimentos: À Fundação de Apoio à Pesquisa e Inovação Tecnológica do Estado de Sergipe (FAPITEC/

$\mathrm{SE})$ e ao Conselho Nacional de Desenvolvimento Científico e Tecnológico (CNPq).
\end{abstract}


logistic regression, to smoke and female sex have been additive impact on stress. The average family income (equal to or above ten minimum wages) and practice regular physical activity have been subtractive impact on stress. We also discussed, besides the models, features of stress measure and differences observed in analysis by linear and logistic regression. At the end, study limitations were highlighted, especially concerning the choice of Faces Scale, and suggestions for further research were also made.

Keywords: Stress, distress, faces scale, sociodemographic profile, health habits.

\section{Estrés y Distrés: Investigación con la Escala de Faces en Aracaju (SE)}

\section{Resumen}

Esta investigación tuvo como objetivo recoger datos a cerca de la percepción de estrés en Aracaju (SE), así como identificar la presencia de distrés, caracterizar los perfiles sociodemográfico y de la salud de la muestra, y mapear las relaciones de estos perfiles con la variabilidad del estrés y distrés. El muestreo se realizó mediante la técnica de punto de flujo, en que participaron 2135 adultos que se encontraban en el principal centro comercial de Aracaju (SE). Se utilizó un cuestionario para la caracterización sociodemográfica y de salud, mientras que el estrés y el distrés fueron medidos utilizando la Escala de Faces (siete puntos). Para el análisis estadístico se estimó el impacto de las variables independientes sobre el estrés por regresión lineal y el distrés con regresión logística. En los resultados, 15,1\% de los participantes mostró distrés y el promedio de la escala fue tres puntos (desviación estándar $=1.5$ ). Las variables ingreso, sexo, tabaquismo y actividad física mostraron significación estadística con el estrés. En los modelos de regresión lineal y logística, tuvieron impacto aditivo sobre el estrés el tabaquismo y sexo, y con impacto sustractivo ingreso familiar promedio (extracto igual o superior a diez salarios mínimos) y práctica de actividad física regular. Se discutieron, además de los modelos, características de la medida del estrés y las diferencias observadas por medio de las regresiones lineal y logística. Al final, se señalaron limitaciones del estudio, especialmente en la elección de la Escala de Faces, como también, se sugirieron nuevas posibilidades de investigación.

Palabras clave: Estrés, distrés, escala de faces, perfil sociodemográfico, hábitos de salud.

O estresse tem sido destacado como importante fator para o desencadeamento de uma série de doenças, tais como a hipertensão, a diabetes e a depressão, entre outras de elevada prevalência (Cohen, Janick-Deverts, \& Miller, 2007). Ele pode atuar como facilitador do desenvolvimento e progressão de diversas formas de adoecimento, com repercussão não apenas no indivíduo, mas também ressoar no entorno social em que a pessoa se insere ou compartilha (Miller, Chen, \& Cole, 2009; Santos, 2010).

Por definição, o estresse é um fenômeno psicossocial com repercussão biológica, que ocorre quando há à percepção de ameaça real ou imaginária que venha a ser interpretada como capaz de causar danos em nível psicológico ou físico de um indivíduo (Cohen et al., 2007; Santos, 2010).
Os alvos primários do estresse são a dinâmica neurofisiológica mobilizada frente às experiências estressoras e as transformações adaptativas ocasionadas pelo enfrentamento da situação estressógena, fatores esses que podem desencadear problemas de saúde e/ou ajustamento social e psicológico em curto, médio ou longo prazo (Gunnar \& Quevedo, 2007). Em suma, o estresse é apreendido como o resultado de uma avaliação cognitiva que desencadeia processos regulatórios nas dinâmicas emocional, fisiológica e comportamental, evocando um estado de resposta adaptativa.

As experiências estressoras compreendem situações que suscitam a necessidade de adaptação, por exemplo, a efetuação de alguma mudança de comportamento, dos hábitos cotidianos ou 
do estilo de vida (Dohrenwend, 2006; Monroe, 2008). Partindo-se para uma análise conjuntural da temática dos estressores, a investigação sobre a probabilidade de ser mais vulnerável ao risco acumulativo de estressores é uma necessidade atual, posto que, assim, seria possível conhecer as características que mais expõem as pessoas aos efeitos adversos do estresse, ou ainda, as protegem de seus efeitos (Schnittker \& McLeod, 2005; Schwartz \& Meyer, 2010).

$\mathrm{O}$ estresse, enquanto processo, trata-se do mecanismo regulatório que organismo e mente ativam diante de uma experiência estressora, buscando a redução da sensação de desconforto, mal-estar ou sofrimento, como fora apontado por Hans Selye, considerado o pai da teoria (Selye, 1993). Porém, por si só, o estresse não é um elemento lesivo, tendo em vista que somente quando a carga estressora supera a capacidade de ajustamento é que o indivíduo passa a sofrer consequências danosas. Do contrário, quando há recursos o suficiente para lidar com o advento do estressor, há a capacidade de retorno ao estado de bem-estar psicológico anterior e à homeostase (McEwen, 2008; Santos, 2010). Como destacado em McEwen e Lasley (2007), é o excesso de exposição ao estresse que gera prejuízos ao organismo, pois, visando à adaptação, são ativados inúmeros recursos neurofisiológicos, hormonais e mentais, que, se agem apenas em curto prazo, favorecem a adaptação; mas, se perduram mobilizados, propiciam o desgaste do próprio organismo e o aparecimento de patologias adaptativas, uma vez que sobrecarregam o funcionamento de diversos sistemas (por exemplo, o cortisol e o sistema imunológico; ver Soares \& Alves, 2006).

Ao se falar em distresse, refere-se ao estado de desgaste do sistema adaptativo, caracterizado por sintomas de depressão, ansiedade e manifestações somáticas, em que os recursos de ajustamento mobilizados pelo indivíduo para lidar com o elemento estressógeno não foram capazes de prover a adaptação, falhando em restabelecer o estado de homeostase física e mental (Drapeau, Marchand, \& Beaulieu-Prévost, 2012). Como consequência, o distresse faz menção à condição de vulnerabilidade do indivíduo ao adoecimen- to derivada do excesso de sobrecarga adaptativa (Feldman, Dunn, Stemke, Bell, \& Greeson, 2014), sendo, também, entendido como sofrimento psicológico provocado pelo estresse e facilitador para o aparecimento de doenças (Anderson et al., 2013; Sklar, Groessl, O'Connell, Davidson, \& Aarons, 2013).

O distresse é considerado um dos indicadores de saúde mental mais importantes para rastreamento de vulnerabilidade ao desenvolvimento de transtornos mentais comuns, a exemplo dos transtornos de ansiedade e depressão (Donker et al., 2010; Drapeau et al., 2012). Isso se dá pelo fato de ser uma resposta adaptativa inespecífica, isto é, surgir em virtude de situações estressoras diversas que afetam o bem-estar físico e psicológico (Beatty \& Lambert, 2013; Dallo, Kindratt, \& Snell, 2013; Zaroff, Davis, Chio, \& Madhavan, 2012).

Para Dallo et al. (2013), o distresse tem recebido pouca atenção nas pesquisas em saúde - embora o volume de pesquisas denote um crescimento progressivo -, dada a quantidade de patologias e psicopatologias que são associadas, seja no desencadeamento, seja no agravamento, ao distresse. Assim, visando a alinhar a presente proposta a essa demanda ainda presente no campo de estudos da saúde, destaca-se que entre os objetos de estudo da psicologia da saúde, o estresse e o distresse figuram como importantes linhas de investigação (Stroebe \& Stroebe, 1995), tendo em vista as comprovações de que seus efeitos sobre a saúde podem ser tanto diretos, alterando o funcionamento neurofisiológico, como indiretos, ao afetar a manifestação de comportamentos preventivos ou influenciar negativamente o progresso, tratamento e recuperação das doenças (Drapeau et al., 2012; McEwen, 2008; Monroe, 2008; Sklar et al., 2013).

A despeito de sua importância, percebe-se que ainda são necessários os estudos que analisem, especificamente, o impacto conjunto de diferentes características sociodemográficas (por exemplo, idade, sexo, dentre outros) e comportamentos de saúde (por exemplo, tabagismo e prática de atividade física) na distribuição social do estresse (Schwartz \& Meyer, 2010). Essa necessidade também se aplica ao contexto brasilei- 
ro das pesquisas sobre este tema, que tendem a privilegiar amostragens de contextos singulares (por exemplo, estudantes universitários), sendo escassos estudos voltados à população em geral de um dado local, cuja proposta seria a da discriminação do efeito conjunto de diversos estressores sobre a adaptação psicológica (Santos, 2010; Sparrenberger Santos, \& Lima, 2003).

Com propostas alinhadas a essa demanda por levantamentos populacionais em território brasileiro, é possível citar como exemplo a pesquisa conduzida por Sparrenberger et al. (2003) no município de Pelotas (RS), em que foi encontrada uma prevalência de $14 \%$ para o distresse. Além dessa, outras pesquisas com objetivo similar foram realizadas na cidade de São Paulo e em Santa Catarina, obtendo-se índices de estresse em $32 \%$ e $13,9 \%$, respectivamente (Barros \& Nahas, 2001; Lipp, Pereira, Floksztrumpf, Muniz, \& Ismael, 1996). Destaca-se, contudo, que os estudos citados foram realizados apenas em duas regiões do país (Sul e Sudeste), e que, apesar de haver informações sobre índices de estresse nessas regiões do Brasil, não foi encontrado, em meio a estudos publicados nas bases de dados Scielo e Pepsic até Dezembro de 2013, pesquisas com essa proposta na região Nordeste.

Vale aqui destacar a medida do estresse com a Escala de Faces. Em geral, escalas desse tipo possuem de cinco a sete faces estilizadas que representam estados emocionais, indo desde o extremo desprazer ao extremo prazer, cujo intuito é revelar a percepção do indivíduo a respeito de um tema em questão. Esse tipo de escala pode ser utilizado como uma medida generalista de bem-estar, direcionada a uma temática em particular (por exemplo, percepção de dor) ou geral (por exemplo, satisfação com a vida), visando a avaliar a autopercepção do indivíduo sobre o assunto abordado (usualmente limitando a um período que compreende a última semana, mês ou ano; McDowell, 2006).

Em relação ao distresse, a Escala de Faces pode ser aplicada sob o princípio da avaliação da reação de ajustamento às adversidades, posto que indivíduos que tenham passado por dificuldades adaptativas frente a situações estressoras tendem a apontar a resultante de suas experiências como sofrimento emocional ou mal-estar psicológico (Drapeau et al., 2012). Logo, ela pode ser vista como uma alternativa prática e simples para rastreamento de distresse, funcionando como um filtro preliminar de possíveis demandas na atenção primária à saúde (McDowell, 2006). A exemplo de aplicações recentes e que detectaram a viabilidade empírica de medidas simples (baseadas em um único item), Harper et al. (2013) utilizaram uma Escala de Faces para avaliar estresse parental em situações de cuidado de filhos com câncer, além de Jacobsen et al. (2005), que aplicaram o termômetro de distresse (uma medida similar) junto a pacientes ambulatoriais com câncer.

No campo do estresse, a proposição de uso de escalas de única medida é interessante a estudos que objetivem levantamentos gerais (screening) da autopercepção da adaptação psicológica a eventos estressores diversos. Logo, por ser considerada uma das mais simples, de fácil e rápida aplicação, torna-se um instrumento viável para estudos com amostragem numerosa, tal qual se propõe nesta investigação. Diante do exposto, esta pesquisa objetivou:

1. Levantar dados a respeito da autopercepção de estresse em Aracaju (SE);

2. Identificar a presença de distresse;

3. Caracterizar o perfil sociodemográfico e hábitos de saúde da amostra; e,

4. Mapear relações desses perfis quanto à variabilidade do estresse e do distresse.

\section{Método}

\section{Participantes}

O presente estudo teve delineamento transversal e participaram 2135 transeuntes adultos que se encontravam no principal centro comercial de Aracaju (SE). A técnica escolhida para amostragem foi por Ponto-de-Fluxo (Hornik \& Ellis, 1988; Samara \& Barros, 2002), que se caracteriza pela obtenção de dados em determinados locais de interesse e grande movimentação de indivíduos alvos do estudo, por meio da estratégia de interceptação sistemática (convite), com ou sem aleatorização, de potenciais participantes. 
A coleta foi realizada no centro comercial de Aracaju (SE), região em que estão instaladas diversas lojas, agências bancárias e outras instituições que motivam grande circulação de pessoas. Dentre 12 esquinas formadas pelo cruzamento de vias públicas que delimitam (aproximadamente) a região do centro comercial, sortearam-se três no chamado "Calçadão" do centro da cidade; um local onde só é permitido o trânsito de pedestres.

A equipe de entrevistadores foi composta por seis estudantes de psicologia, previamente treinados e supervisionados, organizados em três duplas, cada uma responsável pela coleta em uma das esquinas selecionadas. Os entrevistadores abordavam indivíduos adultos que estivessem transitando no local pré-determinado, apresentando-lhes o tema e objetivos da pesquisa. Havendo a aceitação em participar, assinava-se o termo de consentimento livre e esclarecido e procedia-se ao preenchimento do questionário. Para o próximo convite, aguardava-se a passagem de outros três indivíduos e era convidado o quarto transeunte. Tal procedimento foi assumido com o intuito de prover uma forma de sistematização adicional à coleta, reforçando a busca pela randomização do procedimento (Hornik \& Ellis, 1988). Caso não houvesse a aceitação, o convite era feito ao seguinte indivíduo, até que se obtivesse a quantidade mínima estipulada por entrevistador (350 indivíduos).

\section{Instrumentos}

Utilizou-se um questionário fechado composto por nove questões, composto pelas variáveis sociodemográficas: sexo (masculino ou feminino), idade (em anos, entre 18 e 65), relacionamento conjugal estável (em relacionamento ou sem relacionamento), escolaridade (até o ensino fundamental, médio e superior), atividade laborativa remunerada (sim e não), renda média familiar nos últimos 3 meses (até 2 salários mínimos, 2 salários, 3 a 5 salários, 5 a 10 salários e acima de 10 salários), local de residência (capital ou interior), zona de moradia (urbana ou rural) e religião (sim ou não).

Além dos dados sociodemográficos, incluiu-se duas questões acerca de hábitos de saúde: tabagismo ( sim ou não) e prática de atividade física regular (sim ou não). Na variável tabagismo se consideraram fumantes os sujeitos que fumaram pelo menos um cigarro por dia há pelo menos um mês (Menezes et al., 2004) e, quanto à atividade física regular, considerava-se inativo (ou sedentário) o indivíduo que não praticava atividades de pequeno esforço físico por pelo menos 30 minutos diários, tal como caminhada livre ou para o trabalho, andar de bicicleta ou atividades similares (Duncan et al., 1993).

A autopercepção de estresse foi mensurada com a Escala de Faces, formada por sete imagens que variam entre a expressão de extrema felicidade (valor 1) à extrema tristeza (valor 7), apontando-se a figura que mais se aproxima da avaliação que o indivíduo faz da sua vida no último ano. As respostas que compreendiam os valores 5, 6 e 7 foram o parâmetro para a presença de distresse (McDowell, 2006). Tal instrumento fora utilizado também no estudo de Sparrenberger et al. (2003), cuja replicação e assunção do mesmo ponto de corte visou à comparabilidade dos achados.

\section{Análise dos Dados}

Os dados foram analisados com o programa SPSS (versão 19.0). Aplicaram-se testes de correlação de Spearman (idade), ANOVA one-way (escolaridade e renda média familiar) e teste $t$ de Student (sexo, relacionamento conjugal estável, local de residência, zona de moradia, ocupação, religião, tabagismo e atividade física). Somados a esses, executaram-se correlações bisseriais por ponto e coeficientes de correlação bisserial, com o intuito de contraprova aos resultados obtidos nas análises bivariadas iniciais. $\mathrm{O}$ nível de significância assumido foi de $p<0,05$ e utilizou-se o critério de Cohen para interpretação do Tamanho de Efeito $(d)$. Após a seleção e ajuste das variáveis para a análise multivariada, realizaram-se procedimentos de regressão linear (método enter), tendo como variável dependente a pontuação na Escala de Faces.

Efetuou-se, ainda, análise de regressão logística [método forward (Wald)] e a variável critério foi o diagnóstico de distresse ( 0 - não e 1 sim). Vale salientar que os indicadores de $O d d s$ Ratio $(O R)$ com valores inferiores a 1 no resulta- 
do da regressão logística foram convertidos por meio da fórmula " $1 /$ valor da $O R$ ", com a intenção de facilitar a interpretação do índice como fator de proteção. Em ambas as regressões, as variáveis que denotaram significância estatística na análise bivariada foram incluídas como preditoras (regressão linear) ou covariantes (regressão logística) nos modelos testados (Tabachnick \& Fidel, 2007).

\section{Resultados}

A distribuição da pontuação na escala de faces e o diagnóstico de distresse estão representados na Figura 1. A média na escala de faces foi de 3,0 pontos, $D P=1,5$, sendo que $15,1 \%(n=322)$ dos participantes pontuaram igual ou acima de 5, indicando a presença de distresse (Figura 1).

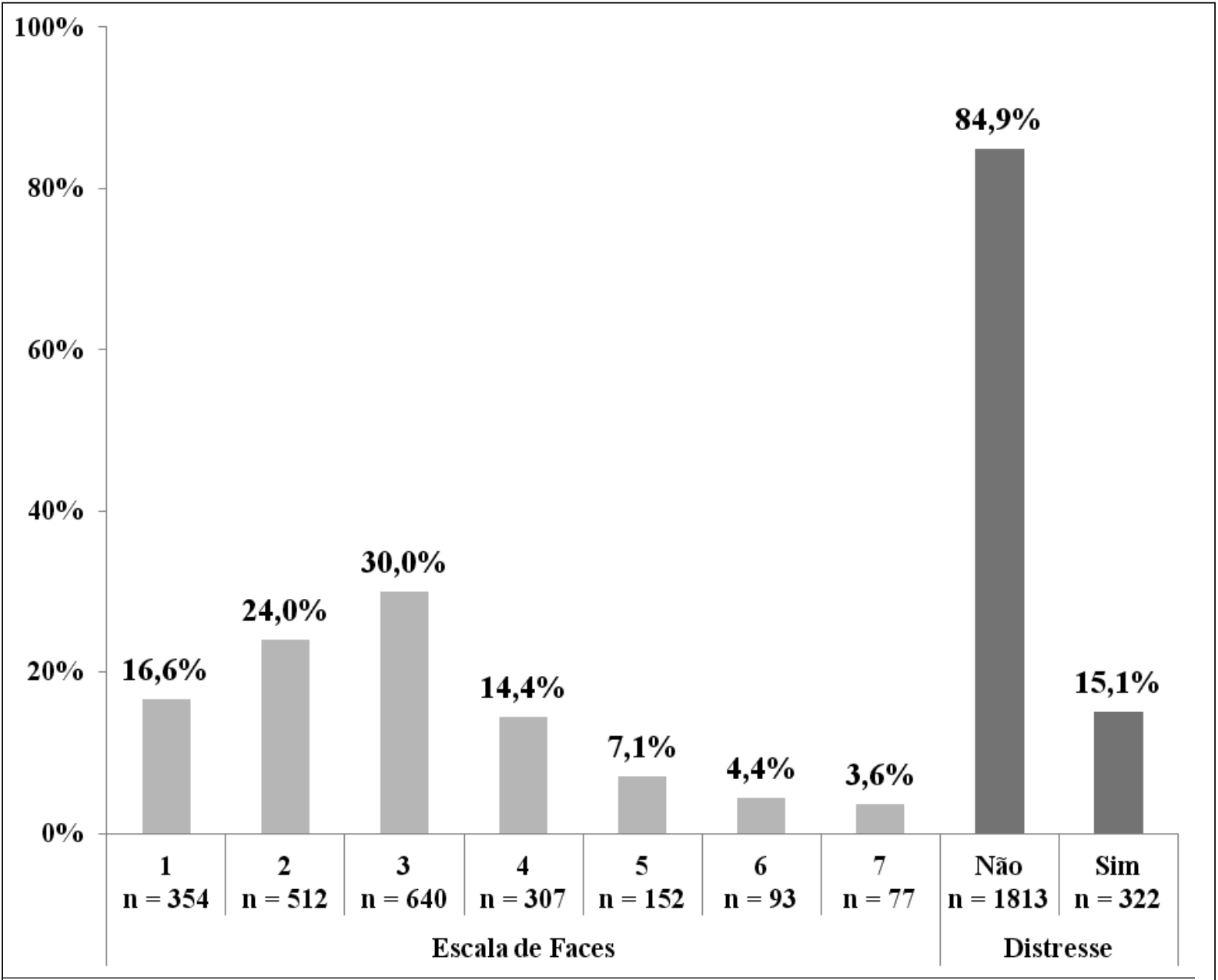

Figura 1. Frequências absoluta e percentual das pontuações na Escala de Faces e do Diagnóstico de Distresse (Aracaju, SE).

O perfil sociodemográfico está descrito na Tabela 1. A média de idade foi de 31,6 anos (DP $=11,81)$, com predomínio do sexo feminino $(51,0 \%)$, pessoas sem relacionamento conjugal estável $(59,6 \%)$, com escolaridade em ensino médio $(58,5 \%)$ e renda familiar entre 3 e 5 salários mínimos (30,7\%). A maioria exercia atividade laborativa $(63,3 \%)$, morava na capital $(69,9 \%)$ e zona urbana $(95,6 \%)$. A maior parte declarou professar religião $(84,6 \%)$, sendo que $12,2 \%$ declararam-se tabagistas e $35,4 \%$ praticavam atividade física regular.

Quanto ao estresse, na análise bivariada se constatou que a idade não indicou correlação estatisticamente significativa $(\rho=0,03 ; p=0,131)$. Com o teste $t$ de Student se verificou que as variáveis relacionamento conjugal estável, $t(2133)$ $=-0,345 ; p=0,730$; local de residência, $t(2133)$ 
Tabela 1

Estatística Descritiva das Variáveis Sociodemográficas e dos Comportamentos de Saúde (Aracaju, SE)

\begin{tabular}{|c|c|c|c|}
\hline Variáveis & & $N(2135)$ & $F(\%)$ \\
\hline \multirow[t]{2}{*}{ Sexo } & Masculino & 1046 & 49,0 \\
\hline & Feminino & 1089 & 51,0 \\
\hline \multirow[t]{2}{*}{ Relacionamento conjugal estável } & Em relacionamento & 862 & 40,4 \\
\hline & Sem relacionamento & 1273 & 59,6 \\
\hline \multirow[t]{3}{*}{ Escolaridade } & Até o fundamental & 506 & 23,7 \\
\hline & Médio & 1248 & 58,5 \\
\hline & Superior & 381 & 17,8 \\
\hline \multirow[t]{5}{*}{ Renda média familiar (em salários mínimos - SM) } & Menor que $2 \mathrm{SM}$ & 507 & 23,7 \\
\hline & $2 \mathrm{SM}$ & 496 & 23,2 \\
\hline & 3 a $5 \mathrm{SM}$ & 655 & 30,7 \\
\hline & 5 a $10 \mathrm{SM}$ & 325 & 15,2 \\
\hline & Acima de $10 \mathrm{SM}$ & 152 & 7,1 \\
\hline \multirow[t]{2}{*}{ Atividade laborativa remunerada } & $\operatorname{Sim}$ & 1358 & 63,3 \\
\hline & Não & 777 & 36,4 \\
\hline \multirow[t]{2}{*}{ Local de residência } & Capital & 1492 & 69,9 \\
\hline & Interior & 643 & 30,1 \\
\hline \multirow[t]{2}{*}{ Zona de moradia } & Rural & 93 & 4,4 \\
\hline & Urbana & 2042 & 95,6 \\
\hline \multirow[t]{2}{*}{ Religião } & $\operatorname{Sim}$ & 1806 & 84,6 \\
\hline & Não & 329 & 15,4 \\
\hline \multirow[t]{2}{*}{ Tabagismo regular } & Sim & 261 & 12,2 \\
\hline & Não & 1874 & 87,8 \\
\hline \multirow[t]{2}{*}{ Atividade física regular } & $\operatorname{Sim}$ & 756 & 35,4 \\
\hline & Não & 1379 & 64,6 \\
\hline
\end{tabular}

$=0,952 ; p=0,341 ;$ zona de moradia, $t(2133)=$ 1,$121 ; p=0,262$ e religião, $t(2133)=-0,419 ; p$ $=0,675$ não apresentaram diferença significativa $(p>0,05)$.

As variáveis que denotaram diferenças estatisticamente significativas com o estresse foram o sexo, $t(2122,4)=2,211 ; p=0,027 ; d=0,13$ : o feminino obteve maior pontuação média de estresse $(M=3,1 ; D P=1,59)$, em comparação ao masculino $(M=2,9 ; D P=1,42)$. Além do sexo, o tabagismo, $t(319,0)=-4,251 ; p<0,001$; $d=0,31$ e a prática regular de atividade física, $t(1694,0)=4,358 ; p<0,001 ; d=0,20$ tiveram diferença, sendo que os indivíduos tabagistas
$(M=3,4 ; D P=1,68)$ e os que não praticavam atividade física $(M=3,1 ; D P=1,56)$ indicaram maiores pontuações na escala de faces, em comparação aos não tabagistas $(M=2,9 ; D P=1,48)$ e os que praticavam atividade física $(M=2,8$; $D P=1,41)$.

Com a ANOVA one-way, encontrou-se que quanto à escolaridade, $F(2,2132)=1,542 ; p=$ 0,214 não houve significância estatística em relação à escala de faces $(p>0,05)$. Quanto à renda média, detectou-se significância estatística, $F(4,2130)=3,890 ; p=0,004$. Com o post-hoc LSD se observou que o estrato com 10 ou mais salários mínimos $(d=0,29)$ exibiu menor pon- 
tuação na escala de faces $(M=2,5 ; D P=1,17)$, quando comparado aos demais grupos: até 2 salários mínimos $(M=3,1 ; D P=1,75), 2$ salários $(M=3,1 ; D P=1,53), 3$ a 5 salários $(M=3,0 ; D P$ $=1,39)$ e 5 a 10 salários $(M=2,9 ; D P=1,44)$.

Como contraprova aos resultados da análise bivariada e para ratificar a escolha das variáveis a serem inseridas na regressão, executou-se testes de correlação bisserial por ponto $\left(r_{p b}\right)$ para as variáveis sexo, tabagismo e atividade física. Com isso, detectou-se que todas as correlações mantiveram-se significativas, com valores de $r_{p b}$ iguais a $0,048(p=0,028)$ para o sexo, $0,101(p<$ $0,001)$ para o tabagismo e $0,091(p=0,007)$ para a atividade física. Com a variável renda média, calculou-se o coeficiente de correlação bisserial $\left(r_{b}\right)$, que foi de $0,126(p<0,001)$. Assim, ten- do sido mantidos os quatro preditores com $p$ -valores menores que 0,05 , eles foram inseridos na modelagem por regressão linear (codificados como dummy).

Ao final da regressão linear, todas as variáveis preditoras permaneceram no modelo, $F(4$, $2130)=12,980 ; R^{2}=0,024 ; p<0,001$ (Tabela $2)$, sendo que os coeficientes de regressão padronizados $(\beta)$ indicaram que o tabagismo teve o maior impacto aditivo na pontuação do estresse $(\beta=0,104)$, seguido do sexo feminino $(\beta=$ $0,043)$. Com impacto subtrativo, a atividade física regular $(\beta=-0,072)$ foi o principal, seguido da renda igual ou acima de 10 salários $(\beta=-0,065)$. A análise de resíduos e ScatterPlots não apontou a existência de condições de inadequação dos resultados obtidos.

Tabela 2

Sumário da Regressão Linear entre Características Sociodemográficas, Hábitos de Saúde e o Estresse (Aracaju, SE)

\begin{tabular}{lccccc}
\hline \multicolumn{1}{c}{ Modelo } & $B$ & SEB & $t$ & $\beta$ & $p$ \\
\hline Constante & 3,103 & 0,050 & 62,044 & - & $<0,001$ \\
Tabagismo & 0,480 & 0,101 & 1,945 & 0,104 & $<0,001$ \\
Atividade física regular (sim) & 0,228 & 0,070 & 4,771 & 0,072 & 0,001 \\
Renda ( $\geq 10$ salários mínimos) & 0,386 & 0,127 & 3,268 & 0,065 & 0,002 \\
Sexo feminino & 0,131 & 0,067 & 3,042 & 0,043 & 0,050 \\
\hline
\end{tabular}

Nota. $R=0,154 ; R^{2}=0,024(p<0,001)$.

Para a regressão logística se utilizou o diagnóstico de distresse como variável critério $(1=$ sim) e foram inseridas como covariantes: tabagismo $(1=\operatorname{sim})$, sexo $(1=$ feminino $)$, prática de atividade física regular $(1=\operatorname{sim})$ e renda média familiar (por extrato, $1=$ sim). O modelo final foi alcançado em quatro passos $(-2 L L$ inicial $=$ $1794,045 ;-2 L L$ final $=1752,996)$, não havendo diferenças significativas entre os modelos esperado e observado [Hosmer e Lemeshow $\left(x^{2}\right)$ $=5,411 ; p=0,492]$. As probabilidades [Odds Ratio (OR)] com significância estatística ( $p<$ $0,001)$ resultaram nos seguintes valores: com probabilidade aditiva, o maior foi o tabagismo $(O R=2,2)$ e em seguida o sexo feminino $(O R=$ 1,6). Com probabilidade subtrativa, a maior foi o extrato de renda de 10 ou mais salários mínimos
$(O R=0,4$; após conversão $O R=2,5)$, seguido da prática de atividade física regular $(O R=0,7$; após conversão $O R=1,4)$. Ao final, a partir da avaliação da tabela de classificação de casos, que compara predições do modelo logístico com os efetivos resultados obtidos, observou-se que $85 \%$ dos casos foram corretamente preditos.

\section{Discussão}

Quanto ao distresse, o índice encontrado nesta pesquisa $(15,1 \%)$ ficou próximo ao obtido em Pelotas (RS) em uma amostra populacional de 3.492 pessoas, cujo índice, também por meio da Escala de Faces e mesmo ponto de corte, foi de 14,0\% (Sparrenberger et al., 2003). A ausência de significância estatística das variáveis ida- 
de e escolaridade em relação ao estresse diferiu dos achados em Pelotas, onde foi encontrada relevância nessas comparações. As características referentes à ocupação, religião, zona de moradia, local de residência, prática de atividade física, tabagismo e relacionamento conjugal não foram avaliadas no referido estudo, portanto, não sendo passíveis de comparação. Por outro lado, a relação detectada com o sexo e a renda acompanharam o estudo em Pelotas, reiterando o impacto significativo dessas variáveis sobre o estresse.

Quanto aos fatores de explicação do estresse observados neste estudo, observou-se que os tabagistas, os que não praticam atividade física, as mulheres e os que tinham renda média menor que 10 salários mínimos exibiram pontuações mais altas na escala de faces e, também, maior probabilidade de comporem o grupo com distresse.

Enquanto fator preditivo, o tabagismo foi a variável de maior impacto $(\beta=0,104)$, com o tabagista tendo probabilidade 2,2 vezes maior que o não tabagista de apresentar distresse. $\mathrm{O}$ consumo de cigarros manteve índice menor do que o nível nacional $(17,5 \%)$ e semelhante ao do Estado de Sergipe (12,1\%; Instituto Brasileiro de Geografia e Estatística [IBGE], 2009), exibindo associação com o estresse e sendo o mais forte preditor. Ressalta-se que, independentemente da sua proporção, o uso de tabaco é um hábito de risco que se apresenta correlacionado a inúmeras doenças, sendo o estresse mecanismo de vulnerabilização que se pode aliar a esta predisponibilidade (Baker, Brandon, \& Chassin 2004).

Assentindo a outros estudiosos, o tabagismo é um hábito que pode estar associado a complexas condições personológicas e sociais que implicam a adição como mecanismo de enfrentamento inadequado para lidar com os eventos estressores (Baker et al., 2004). Assim, depreende-se que o tabagista pode ser um sujeito que compõe um grupo mais vulnerável ao estresse, e também indica pertencer a uma parcela populacional que requer intervenções no sentido de modificação na forma de manejar estressores e o próprio estresse.

Em direção contrária ao tabagismo, a atividade física se associou negativamente com o estresse e apareceu como fator protetor. A prática regular de atividades físicas foi o principal preditor negativo do estresse $(\beta=-0,072)$ e, quanto ao distresse, um praticante de atividade física exibiu 1,4 vezes menos chances de apresentar distresse $(O R=1,4)$, em comparação aos que não praticavam. Cientificamente é quase consensual o papel da atividade física regular como uma das chaves para a promoção e manutenção da saúde (Buss, 2000), o que foi reforçado por este estudo, visto o impacto subtrativo sobre o estresse.

O sexo feminino se destacou como variável associada positivamente com o estresse, demonstrando impacto aditivo sobre a sua predição $(\beta=0,043)$. Noutra leitura, as mulheres denotaram uma probabilidade 1,6 vezes maior que os homens de apresentarem distresse. Corroborando este achado, na literatura é encontrada a associação das mulheres a mais altos índices de estresse em contextos nacionais (Barros \& Nahas, 2001) e internacionais (Denton, Prus, \& Walters, 2008). Inclusive, estudos também mostram que as mulheres exibem picos hormonais com maior severidade e restabelecimento mais lento do padrão metabólico, o que reforça a hipótese de que elas são mais vulneráveis a perturbações sistêmicas de saúde por desequilíbrio hormonal duradouro, principalmente diante de estresse crônico (Nepomnaschy, Sheiner, Mastorakos, \& Arck, 2007; Otte et al., 2005).

Outra hipótese para explicar a maior prevalência entre as mulheres se deve ao fato de que elas indicam com maior facilidade a presença de sintomas de estresse em instrumentos de autorrelato ou mantêm maior nível de vigilância sobre a própria saúde em comparação aos homens (Denton et al., 2008). Todavia, a constância dos achados suscita a conclusão de que os estressores aos quais as mulheres estão submetidas implicam marcantes prejuízos acumulativos para a saúde física e mental. Logo, acima da própria condição de estarem mais atentas às alterações de seus estados físicos e psíquicos, percebe-se que a estrutura social ainda responde por um seletivo impacto sobre a saúde das mulheres.

A renda média familiar apresentou diferença sobre o estresse apenas entre os que afirmaram ganhar 10 salários mínimos ou mais $(\beta=$ 
$-0,065)$, sendo 2,5 vezes menor a chance desse grupo exibir distresse $(O R=2,5)$. Na literatura se observa, com frequência, que há uma relação preditiva entre estresse e renda: quanto menor a renda, maior a probabilidade de possuir altas pontuações nas escalas de estresse (Wilkinson \& Marmot, 2003) Tal como afirmaram Siegrist e Marmot (2004), pode-se apreender também que a cada passo na redução do status socioeconômico, maior a vulnerabilidade dos indivíduos. Por outro lado, o que se espera é que com o crescimento da renda seja reduzida a exposição a contextos estressógenos, uma vez que melhores recursos materiais minimizam a existência de estressores associados à baixa qualidade de vida e a exposição à mazelas sociais ligadas à pobreza (Orpana, Lemyre, \& Gravel, 2009). Com efeito, acredita-se que com a elevação da renda é possível minimizar o impacto do estresse, indo além do próprio status social, pois isso reforça a compreensão de que a melhoria nas condições socioeconômicas de uma população atua como peça-chave para atingir melhores níveis de saúde em geral (Schnittker \& McLeod, 2005).

Para Orpana et al. (2009), a renda seria um dos principais fatores para explicar a causação social dos piores níveis de saúde mental, inclusive o estresse, nas camadas mais pobres da população. Logo, embora a renda não reflita totalmente o impacto do status socioeconômico sobre o estresse, ela representa uma importante variável na quantificação da exposição e na ativação dos recursos psicossociais de adaptação (Marmot, Siegrist, \& Theorell, 2006; Wilkinson, 2006).

Uma das hipóteses de explicação da relação entre o estresse e a renda se dá a partir da noção de privação relativa. Esse conceito trata de um processo pelo qual a pessoa compara sua vida a de outros que considera como pares (reais ou ideais) em um dado contexto social, o que ocorre de acordo com sua percepção de posição social subjetiva (Marmot et al., 2006; Yngwe, Fritzell, Lundberg, Diderichsen, \& Burström, 2003). Nessa lógica, a renda poderia funcionar como um moderador do impacto da posição social percebida sobre o estresse, por ela surgir como um parâmetro de avaliação da privação relativa (Pham-Kanter, 2009; Sakurai, Kawakami, Yamaoka, Ishikawa, \& Hashimoto, 2010).
Todavia, entende-se que futuramente devam ser conduzidos estudos com a finalidade específica da testagem dessa hipótese, posto que a presente pesquisa não contemplou avaliar essa relação.

No que se refere ao tamanho do efeito das variáveis sobre o estresse, observou-se que, em geral, foram pequenos, com destaque para o sexo, que exibiu o menor efeito $(d=0,13)$, e o tabagismo, que foi o maior obtido $(d=0,31)$. A medida de tamanho de efeito reflete a magnitude da relação entre as variáveis (Conboy, 2003), sendo recomendável sua utilização como parâmetro de avaliação do $p$-valor, pois esse tende a ser sensível a alguns vieses, especialmente o criado pelo tamanho da amostra (Lindenau \& Guimarães, 2012).

Como pontuado por Conboy (2003), a interpretação do tamanho de efeito é relativa, já que depende da proposta do estudo e como a sua determinação contribui para o fenômeno em questão. No presente caso, entende-se que há relevância teórica e empírica para o cálculo e relato do tamanho de efeito. Quanto à relevância teórica, ainda que o valor obtido para o sexo, por exemplo, tenha sido pequeno, inúmeros estudos reiteram a existência de diferenças de sexo em relação ao estresse, mas não são descritos, comumente, os tamanhos de efeito. Tal lógica de pertinência também se aplica às demais variáveis (renda, tabagismo e atividade física), pois traz esclarecimentos adicionais para ponderação dos resultados deste e de outros trabalhos.

A respeito da relevância empírica, pontuou-se aqui a lacuna de estudos populacionais no tema abordado, principalmente na proposta de rastreamento, o que faz com que a identificação dos tamanhos de efeito funcione, para além do debate dos dados da própria pesquisa, como parâmetro para demais estudos. Considerando, enfim, que embora seja aconselhado que sempre se descreva o tamanho de efeito nas pesquisas, nem sempre se encontra tais informações (Conboy, 2003; Lindenau \& Guimarães, 2012) e essa é uma justificativa para o relato desses índices na presente investigação.

Quanto ao impacto das variáveis na explicação do estresse (com a regressão linear) e do distresse (com a regressão logística), encontrou-se resultados que merecem atenção, principalmente 
pelas diferenças nos produtos das análises. No primeiro caso, a variância total explicada foi de $2,4 \%\left(R^{2}=0,024\right)$, que pode ser considerada muito pequena, enquanto que na logística a capacidade de explicação do modelo foi elevada, com $85 \%$ de predições corretas. Ainda que por critérios nem sempre diretamente comparáveis, essa discrepância pode ser avaliada à luz da proposta da pesquisa.

Por ter privilegiado a análise de variáveis sociodemográficas e apenas dois hábitos de saúde, parece que a regressão linear evidenciou uma elevada heterogeneidade quanto à exposição aos fatores estressógenos. Supõe-se que isso se explica pelo fato de que, em virtude do delineamento amostral, não se determinou um contexto em comum para a avaliação do estresse (por exemplo, o estresse entre empregados de um setor em uma empresa). Ou seja, avaliou-se apenas a sua variabilidade, independentemente dos estressores ou outras variáveis que também incidem sobre esse fenômeno (por exemplo, as estratégias de enfrentamento). Por outro lado, ao considerarmos o resultado da regressão logística, o percentual de valores corretamente preditos pelo modelo mostrou-se satisfatório, já que conseguiu identificar uma maioria expressiva de casos em que efetivamente o indivíduo estava no estrato com distresse.

Com efeito, embora se tenha utilizado o mesmo instrumento e as técnicas sejam, em alguma medida, similares, observou-se que critérios de análise distintos produziram importantes diferenças na forma de entender a robustez de cada modelagem. Vale apontar que, em comparação à regressão linear, a logística é um método de análise mais flexível, que busca estabelecer as chances de cada caso se situarem em uma das categorias da variável critério (uma condição dicotômica, de desfecho binário). Porém, na regressão linear, a descrição das relações entre as variáveis independentes e a dependente é um procedimento mais sensível à variabilidade dos dados, pois é calculada, ponto-a-ponto, a distância de cada observação para a reta de regressão (Tabachnick \& Fidel, 2007).

Desse modo, entende-se que ainda que a restrição na escolha de preditores pareça ter subestimado ou não apreendido a variabilidade do estresse (o que ocorreu na regressão linear), a seleção desses mesmos preditores para a estimação do desfecho (a regressão logística) exibiu indícios de validade quanto à capacidade de aspectos sociodemográficos e hábitos de saúde preverem a maior chance de estar entre os que apresentam distresse. Percebe-se, então, que a escolha da técnica, combinada com o desenho do estudo, denota significativo impacto nas conclusões. Em outras palavras, é um dado requer atenção na condução de estudos similares.

Como limitações da pesquisa, cabe fazer algumas pontuações e acentuar as ressalvas quanto aos achados da investigação. A principal delas se trata da escolha do instrumento. Embora prática e de fácil medida, a Escala de Faces ainda não permite um diagnóstico conclusivo sobre o estresse ou o distresse. Considerando que apenas dois estudos realizaram sua aplicação em âmbito nacional com esse intuito (o presente trabalho e o de Sparrenberger et al., 2003), há a necessidade de pesquisas que determinem suas propriedades psicométricas para este contexto, o que fora feito em outros locais e que têm incentivado a testagem de medidas de um único item para rastreamento de mal-estar subjetivo e distresse (Frie, Meulen, \& Black, 2012; Jacobsen et al., 2005; McDowell, 2006).

Interessa pontuar que existe discussão quanto à capacidade de medidas desse tipo conseguir refletir uma variável latente ou condição clínica com a especificidade e sensibilidade necessárias ao diagnóstico (McDowell, 2006; Mitchell \& Coyne, 2007), havendo estudos que encontram validade em tal tentativa (Frie et al., 2012; Jacobsen et al., 2005; Watkins, Daniels, Jack, Dickinson, \& Broeck, 2001, por exemplo) e outros que a refutam (Avasarala, Cross, \& Trinkaus, 2003; Mitchell \& Coyne, 2007, por exemplo). De qualquer modo, visto que esta pesquisa não propôs testar a sua validade e que assumiu como viável a utilização apenas da Escala de Faces, julga-se pertinente que próximos estudos verifiquem esses critérios. Destaca-se esse aspecto pois se entende como necessária a busca por instrumentos simples e, sobretudo, confiáveis para mensuração rápida do distresse, o que é útil como um panorama inicial de trabalho, por exemplo, na realização de levantamentos do estado geral de saúde de 
uma população, com o foco dirigido para a atenção primária à saúde.

Salienta-se que a utilização da Escala de Faces, nesta pesquisa, voltou-se para a obtenção de um retrato panorâmico, servindo, prioritariamente, para se conhecer um perfil geral da autopercepção dos indivíduos quanto a suas experiências cotidianas, permitindo identificar, de modo preliminar, a ocorrência de sofrimento psicológico. Logo, embora tenha se aplicado a escala para a obtenção desse screen, incentiva-se a realização de estudos que façam uso de instrumentos específicos para o distresse, visando a uma análise mais aprofundada, a exemplo da K10 (Drapeau et al., 2012), e que também busquem confirmar a validade de medida da Escala de Faces no Brasil, tal qual Decat, Laros e Araújo (2009) fizeram com o termômetro de distresse (uma medida similar) junto a pacientes oncológicos.

Outra limitação do estudo se refere ao fato de não terem sido cotejadas demais condições biopsicossociais endógenas e exógenas relevantes, tais como mapeamento de estressores, medida de variáveis clínicas ligadas à saúde (por exemplo, pressão arterial e cortisol) e percepção de controlabilidade dos estressores, condições estas que poderiam fornecer um modelo explicativo com maior poder de predição.

Em síntese, visto que os objetivos desta pesquisa se restringiram à identificação do impacto de características sociodemográficas e hábitos de saúde sobre o estresse e o distresse, a escolha do instrumento, a ausência de variáveis clínicas e o não cotejamento dos estressores limitam a generalização destes resultados para outros contextos estressógenos possíveis. Recomenda-se, então, que futuros estudos insiram em seu delineamento a investigação de variáveis relacionadas à saúde física e psicológica, além do próprio enfrentamento das adversidades, evidenciando o efeito de situações específicas sobre o distresse e conhecendo melhor o processo de adaptação ao estresse.

\section{Referências}

Anderson, T. M., Sunderland, M., Andrews, G., Titov, N., Dear, B. F., \& Sachdev, P. S. (2013). The 10item Kessler Psychological Distress Scale (K10) as a screening instrument in older individuals. The American Journal of Geriatric Psychiatry, 21, 596-606. doi:10.1016/j.jagp.2013.01.009

Avasarala, J. R., Cross, A. H., \& Trinkaus, K. (2003). Comparative assessment of Yale single question and Beck Depression Inventory scale in screening for depression in multiple sclerosis. Multiple Sclerosis Journal, 9, 307-310. doi:10.1191/1352458503ms900oa

Baker, T. B., Brandon, T. H., \& Chassin, L. (2004). Motivational influences on cigarette smoking. Annual Review of Psychology, 55, 463-491.

Barros, M. V. G., \& Nahas, M. V. (2001). Comportamentos de risco, auto-avaliação do nível de saúde e percepção de estresse entre trabalhadores da indústria. Revista de Saúde Pública, 35, 554563. doi:10.1590/S0034-89102001000600009

Beatty, L., \& Lambert, S. (2013). A systematic review of internet-based self-help therapeutic interventions to improve distress and disease-control among adults with chronic health conditions. Clinical Psychology Review, 33, 609-622. doi:10.1016/j.cpr.2013.03.004

Buss, P. M. (2000). Promoção da saúde e qualidade de vida. Ciência e Saúde Coletiva, 5, 163-177.

Cohen, S., Janick-Deverts, D., \& Miller, G. E. (2007). Psychological stress and disease. JAMA, 298, 1685-1687.

Conboy, J. E. (2003). Algumas medidas típicas univariadas da magnitude do efeito. Análise Psicológica, 2, 145-158.

Dallo, F. J., Kindratt, T. B., \& Snell, T. (2013). Serious psychological distress among non-Hispanic whites in the United States: The importance of nativity status and region of birth. Social Psychiatry and Psychiatric Epidemiology, 48, 1923-1930. doi:10.1007/s00127-013-0703-1

Decat, C. S., Laros, J. A., \& Araujo, T. C. C. F. (2009). Termômetro de distress: Validação de um instrumento breve para avaliação diagnóstica de pacientes oncológicos. Psico-USF, 14, 253-260. doi:10.1590/S1413-82712009000300002

Denton, M., Prus, S., \& Walters, V. (2008). Gender differences in health: A Canadian study of the psychosocial, structural and behavioral determinants of health. Social Science and Medicine, 58, 2585-2600. doi:10.1016/j. socscimed.2003.09.008

Dohrenwend, B. P. (2006). Inventorying stressful life events as risk factors for psychopathology: To- 
ward resolution of the problem of intracategory variability. Psychological Bulletin, 132, 477-495.

Donker, T., Comijs, H., Cuijpers, P., Terluin, B., Nolen, W., Zitman, F., \& Penninx, B. (2010). The validity of the Dutch K10 and extended K10 screening scales for depressive and anxiety disorders. Psychiatry Research, 176, 45-50. doi:10.1016/j.psychres.2009.01.012

Drapeau, A., Marchand, A., \& Beaulieu-Prévost, D. (2012). Epidemiology of psychological distress. In L. Labate (Ed.), Mental illnesses: Understanding, prediction and control, (pp. 105-134). Rijeka, Croatia: InTech.

Duncan, B. B., Schimidt, M. I., Polanczyc, C. A., Hormrich, C. S., Rosa, R. S., \& Achutti, A. C. (1993). Risk factors for non-communicable diseases in a metropolitan area in the south of Brazil: Prevalence and simultanely. Revista de Saúde Publica, 27, 43-48.

Feldman, G., Dunn, E., Stemke, C., Bell, K., \& Greeson, J. (2014). Mindfulness and rumination as predictors of persistence with a distress tolerance task. Personality and Individual Differences, 56, 154-158. doi:10.1016/j.paid.2013.08.040

Frie, K. G., Meulen J., \& Black, N. (2012). Single item on patients' satisfaction with condition provided additional insight into impact of surgery. Journal of Clinical Epidemiology, 65, 619-626. doi:10.1016/j.jclinepi.2011.12.001

Gunnar, M., \& Quevedo, K. (2007). The neurobiology of stress and development. Annual Review of Psychology, 58, 145-173. doi:10.1146/annurev. psych.58.110405.085605

Harper, F. W. K., Peterson, A. M., Uphold, H., Albrecht, T. L., Taub, J. W., Orom, H., ...Penner, L. A. (2013). Longitudinal study of parent caregiving self-efficacy and parent stress reactions with pediatric cancer treatment procedures. Psycho-Oncology, 22, 1658-1664. doi:10.1002/ pon.3199

Hornik, J., \& Ellis, S. (1988). Strategies to secure compliance for a mall intercept interview. $\mathrm{Pu}$ blic Opinion Quarterly, 52, 539-551.

Instituto Brasileiro de Geografia e Estatística. (2009). Pesquisa Nacional do Tabagismo (PETab): Suplemento saúde da pesquisa nacional por amostra de domicílios - 2008. Brasília, DF: Autor.

Jacobsen, P. B., Donovan, K. A., Trask, P. C., Fleishman, S. B., Zabora, J., Baker, F., \& Holland, J. C. (2005). Screening for psychologic distress in ambulatory cancer patients. Cancer, 103, 14941502. doi:10.1002/cncr.20940

Lindenau, J. D., \& Guimarães, L. S. P. (2012). Calculando o tamanho do efeito no SPSS. Revista HCPA, 32, 363-381.

Lipp, M. E. N., Pereira, I. C., Floksztrumpf, C., Muniz, F., \& Ismael, S. C. (1996). Incidência de stress e hipertensão na população de São Paulo. In Anais do simpósio sobre stress e suas implicações: Um encontro internacional (p. 123). Campinas, SP: Associação Brasileira de Estress.

Marmot, M., Siegrist, J., \& Theorell, T. (2006). Health and the psychosocial environment at work. In M. Marmot \& R. G. Wilkinson (Eds.), Social determinants of health (pp. 78-96). New York: Oxford.

McDowell, I. (2006). Measuring health: A guide to rating scales and questionnaires ( $3^{\text {rd }}$ ed.). New York: Oxford University Press.

McEwen, B. S. (2008). Central effects of stress hormones in health and disease: Understanding the protective and damaging effects of stress and stress mediators. European Journal of Pharmacology, 583, 174-185. doi:10.1016/j. ejphar.2007.11.071

McEwen, B. S., \& Lasley, E. N. (2007). Allostatic load: When protection gives way to damage. In A. Monat, R. S. Lazarus, \& G. Reevy (Eds.), The Praeger handbook on stress and coping (pp. 99111). London: Praeger.

Menezes, A. M. B., Hallal, P. C., Silva, F., Souza, M., Paiva, L., D’Àvila, A., ...Horta, B. L. (2004). Tabagismo em estudantes de medicina: Tendências temporais e fatores associados. Jornal Brasileiro de Pneumologia, 30, 223-228.

Miller, G., Chen, E., \& Cole, S. W. (2009). Health psychology: Developing biologically plausible models linking the social world and physical health. Annual Review of Psychology, 60, 501-524. doi:10.1146/annurev. psych.60.110707.163551

Mitchell, A. J., \&Coyne, J. C. (2007). Do ultra-short screening instruments accurately detect depression in primary care? A pooled analysis and meta-analysis of 22 studies. British Journal of General Practice, 57, 144-151.

Monroe, S. M. (2008). Modern approaches to conceptualizing and measuring human life stress. Annual Review of Clinical Psychology, 4, 33-52. doi:10.1146/annurev.clinpsy.4.022007.141207 
Nepomnaschy, P. A., Sheiner, E., Mastorakos, G., \& Arck, P. C. (2007). Stress, immune function, and women's reproduction. Annals of the New York Academy of Sciences, 1113, 350-364.

Orpana, H., Lemyre, L., \& Gravel, R. (2009). Income and psychological distress: The role of the social environment. Statistics Canada: Health Reports, 20, 21-28.

Otte, C., Hart, S., Neylan, T. C., Marmar, C. R., Yaffe, K., \& Mohr, D. C. (2005). A meta-analysis of cortisol response to challenge in human aging: Importance of gender. Psychoneuroendocrinology, 30, 80-91.

Pham-Kanter, G. (2009). Social comparisons and health: Can having richer friends and neighbors make you sick? Social Science \& Medicine, 69 , 335-344. doi:10.1016/j.socscimed.2009.05.017

Sakurai, K., Kawakami, N., Yamaoka, K., Ishikawa, H., \& Hashimoto, H. (2010). The impact of subjective and objective social status on psychological distress among men and women in Japan. Social Science \& Medicine, 70, 1832-1839. doi:10.1016/j.socscimed.2010.01.019

Samara, B. S., \& Barros, J. C. (2002). Pesquisa de marketing: Conceitos e metodologia. São Paulo, SP: Pearson.

Santos, A. F. (2010). Determinantes psicossociais da capacidade adaptativa: Um modelo teórico para o estresse (Tese de doutorado, Universidade Federal da Bahia, Salvador, BA, Brasil).

Schnittker, J., \& McLeod, J. D. (2005). The social psychology of health disparities. Annual Review of Sociology, 31, 75-103. doi:10.1146/annurev. soc. 30.012703 .110622

Schwartz, S., \& Meyer, I. H. (2010). Mental health disparities research: The impact of within and between group analyses on tests of social stress hypotheses. Social Science \& Medicine, 70, 11111118. doi:10.1016/j.socscimed.2009.11.032

Selye, H. (1993). History and present status of the stress concept. In L. Goldberger \& S. Breznitz (Eds.), Handbook of stress: Theoretical and clinical aspects ( $2^{\text {nd }}$ ed., pp. 7-17). New York: Free Press.

Siegrist, J., \& Marmot, M. (2004). Health inequalities and the psychosocial environment: Two scientific challenges. Social Science and Medicine, 58(8), 1463-1473.
Sklar, M., Groessl, E. J., O'Connell, M., Davidson, L., \& Aarons, G. A. (2013). Instruments for measuring mental health recovery: A systematic review. Clinical Psychology Review, 33, 10821095. doi:10.1016/j.cpr.2013.08.002

Soares, A. J. A., \& Alves, M. G. P. (2006). Cortisol como variável em psicologia da saúde. Psicologia, Saúde \& Doenças, 7, 165-177.

Sparrenberger, F., Santos, I., \& Lima, R. C. (2003). Epidemiologia do distress psicológico: Estudo transversal de base populacional. Revista de Saúde Pública, 37, 434-439. doi:10.1590/ S0034-89102003000400007

Stroebe, W., \& Stroebe, M. (1995). Psicologia Social e saúde. Lisboa, Portugal: Instituto Piaget.

Tabachnick, B. G., \& Fidel, L. S. (2007). Using multivariate statistics $\left(5^{\text {th }}\right.$ ed.). New York: Harper Collins.

Watkins, C., Daniels, L., Jack, C., Dickinson, H., \& Broeck, M. S. O. (2001). Accuracy of a single question in screening for depression in a cohort of patients after stroke: comparative study. British Medical Journal, 323, 1159. doi:10.1136/ bmj.323.7322.1159

Wilkinson, R., \& Marmot, M. (2003). Social determinants of health: Solid facts. Copenhagen, Denmark: World Health Organization Europe.

Wilkinson, R. G. (2006). Ourselves and others - for better or worse: Social vulnerability and inequality. In M. Marmot \& R. G. Wilkinson (Eds.), Social determinants of health (pp. 341-358). New York: Oxford.

Yngwe, M. A., Fritzell, J., Lundberg, O., Diderichsen, F., \& Burström, B. (2003). Exploring relative deprivation: Is social comparison a mechanism in the relation between income and health? Social Science and Medicine, 57, 1463-1473. doi:10.1016/S0277-9536(02)00541-5

Zaroff, C. M., Davis, J. M., Chio, P. H., \& Madhavan, D. (2012). Somatic presentation of distress in China. Australian and New Zealand Journal of Psychiatry, 46, 1053-1057. 